\title{
Salivary levels of Interleukin-6, Interleukin-8 and Tumor Necrosis Factor-alpha in Smokers Aged 35-46 Years with Dental Caries Disease
}

\author{
Bayan Jebur Hussein ${ }^{1}$, Hawraa Noori Atallah ${ }^{2}$, Najlaa Abdulameer Ali AL-Dahhan ${ }^{3}$ \\ ${ }^{1}$ Lecturer, Department of Oral Pathology, College of Dentistry, University of Kufa, Al-Najaf, Iraq, ${ }^{2}$ Lecturer, \\ Department of Oral and Maxillofacial Surgery, College of Dentistry, University of Kufa, Al-Najaf, Iraq, ${ }^{3}$ Assist. \\ Prof., Department of Basic Science, College of Dentistry, University of Kufa, Al-Najaf, Iraq
}

\begin{abstract}
Introduction: Dental caries is one of the most common infectious diseases prevalent in the oral cavity, and it is an inflammatory disease with a multi-factor etiology. IL-6, IL-8, and TNF- $\alpha$ are two types of cytokines that are major mediators of acute inflammation, and are necessary for the development of specific immune responses.
\end{abstract}

Objective: The present study aimed to assess the level of pro-inflammatory cytokines (IL-6,IL-8, and TNF- $\alpha$ ) in the saliva of smokers with dental caries and the control (non-smokers) according to different age, BMI, and sex.

Materials and Method: Whole saliva from 32 smokers aged 35-46 years with dental caries and 16 healthy subjects, to measure IL-6,IL-8, and TNF- $\alpha$ levels by enzyme immunoassay (ELISA).

Results: The mean BMI, age, and sex between the experimental group and the control group were similar, were not statistically significant $(\mathrm{p}=0.466, \mathrm{p}=0.240, \mathrm{p}=0.455)$, respectively while, there were a significant difference in CRP between the two groups ( $\mathrm{p}=0.037$ ). Concentrations of salivary IL-6, IL- 8 and TNF- $\alpha$ were not affected by periodontal health. No statistically significant difference in periodontal health, assessed by CPITN, were observed between the groups $(\mathrm{p}=0.413)$. Salivary levels of IL- 6 and IL- 8 were significantly higher in smokers with dental caries compared with control $(p<0.05)$. Salivary levels of IL- 6 and IL- 8 were increased significantly in smokers with dental caries compared with control $(p<0.05)$. No significant differences in salivary TNF- $\alpha$ in dental caries patients compared to control $(\mathrm{p}=0.063)$. The correlation between TNF- $\alpha$, CRP, age, sex, and BMI with IL- 8 were positive and statistically significant $(\mathrm{p}=0.01$, $\mathrm{p}=0.036, \mathrm{p}=0.009, \mathrm{p}=0.007$, and $\mathrm{p}=0.005$ ), respectively. There was no significant correlation between IL-6 and TNF- $\alpha$ cytokines and other parameters, except for a positive association with CRP levels $(\mathrm{p}=0.006$, $\mathrm{p}=0.043)$, respectively.

Conclusions: These data indicate links between production of tumor necrosis factor-alpha (TNF- $\alpha$ ), interleukin-6 (IL-6), and Interleukin-8 (IL-8)in smokers' saliva and dental caries disease. Also,the existence of a statistically significant positive correlation between TNF- $\alpha$,age, gender, and BMI with IL-8.

Keywords: Dentalcaries, saliva, inflammation, interleukin-6, interleukin-8, tumour necrosis factor.

\section{Introduction}

Dental caries is a multifactorial infectious disease, which is determined by the dynamic balance between pathological and protective factors ${ }^{(1)}$. It leads to localized destruction of delicate hard tooth tissues by acidic byproducts resulting from bacterial fermentation of dietary carbohydrates ${ }^{(2)}$.

CRP is a protein that is synthesized in hepatocytes, primarily under stimulation of IL- 6 and IL- 1 in the acute- 
phase, and belongs to the pentraxin family, Its levels are elevated in cases of inflammation, injury or infection ${ }^{(3)}$.

Cytokines play a major role in modulating the immune response, and are either proinflammatory (IL1, IL-8, IL-6, TNF- $\alpha$ and TGF- $\beta$ ), or antiinflammatory (IL-2, IL-4, IL-10, IL-12, and IFN- $\gamma$ ). The nucleus of any cell has the ability to secrete cytokines, but its main source is macrophages and helper $\mathrm{T}$ cells ${ }^{(4)}$. Among the cytokines, interleukins have a crucial function and are implicated in oral cancer.

IL-6 is synthesized in acute inflammatory responses that contribute to host defense. It is involved in the processes of controlling the immune response, inflammation, hematopoiesis, and tumorigenesis. Elevated IL-6 levels may lead to disturbances in immune responses. IL-6 is involved in the regulation of tracking of lymphocytes through the lymph node after developmental stimulation ${ }^{(5)}$. Gabayn found that IL- 6 contributes to the induction of the transition from acute to chronic inflammation through secretion of the monocyte chemoattractant protein-1 $(\mathrm{MCP}-1)^{(6)}$.

IL-8 is an inflammatory stimulant cytokine, and is considered the prototype molecule in the chemokine class. IL-8 also plays an important role in the acute inflammatory response and persists for a relatively long time at the site of inflammation ${ }^{(3)}$. IL-8 release from macrophages and neutrophils is activated by NF- $\mathrm{kB}$.Its expression is modified by other different stimuli, such as hypoxia,inflammation or steroidhormones. IL-8 binds to CRCX-1 and CRCX-2 receptors that are identified on both inflammatory cells from tumor-associated infiltrate and tumour cells ${ }^{(7)}$.

TNF- $\alpha$ is aprotein with a role in host defense and in warning responses. It promotes cell proliferation in the white blood cell molecules adhere to endothelial cells and activate phagocytic killing mechanisms. Besides cytostatic and cytotoxic affecting neoplastic cells $^{(8,9)}$. TNF- $\alpha$ involves nuclear factor kappa B(NF$\kappa \mathrm{B})$, caspase cascades, transcription factors and activating protein1(AP-1), and participation in signal regulation, inflammation, cell growth,and death ${ }^{(3)}$. StJohn et al. indicated that IL-8, IL- 6 , and TNF- $\alpha$ were proven to be overexpressed in $\operatorname{OSCC}^{(10)}$. The present study aimed to evaluate the levels of pro-inflammatory cytokines(IL-6, IL- 8 , and TNF- $\alpha$ ) in the saliva of smokers with dental caries aged between 35-46 years and their relationship withBMI, age, sex and their correlation with each other.

\section{Materials and Method}

Study Population: Forty-eight subjectes their ages ranged from 35-46 years, were divided into two groups, the first group included 16 healthyand non-smoking (10 males and 6 females) as controls,while the second group included 32 smokers(18 males and 14 females) with dental caries enrolled at the Clinical Center at the College of Dentistry at Basra University. From January 2019 to September 2019,they were diagnosed under our supervision. Clinical evaluation procedures e.g. examination of teeth, periodontal, oral mucosal status,assessment of malocclusion and collection of saliva samples. The body mass index (BMI) of each individual was calculated in a astandard manner. Informed consent was obtained from each participant prior to sample collection.

Salivary Samples Collection: Saliva was collected from patient and controls group by a standard method. After the participants were asked to refrain from eating, chewing and drinking at least one hour before the collection. Saliva samples were collected between 09:0011:00 AM.Saliva was collected for $10 \mathrm{~min}$ in titration tubes by drooling method ${ }^{(11)}$. The saliva samples were homogenized and clarified by centrifugation at $10000 \mathrm{~g}$ for 15 min at $4{ }^{\circ} \mathrm{C}$. All samples were stored at $-70{ }^{\circ} \mathrm{C}$ for analysis.

Detection of Salivary Levels of IL-6, IL-8 and TNF- $\boldsymbol{\alpha}$. After collecting salivary samples from nonsmoking(healthy subjectes), and smokin 'dental caries patients, specific cytokines IL-6, IL-8, TNF- $\alpha$ and C-reactive protein (CRP) were measured by human IL6 , IL-8, TNF- $\alpha$ and CRP ELISA kit(Elabscience, Korea). In order to assess the impact of periodontal disease on levels of salivary cytokines,Community Periodontal Index of Treatment Needs was measured in each patient after the collection of saliva ${ }^{(12)}$. Patients with chronic inflammatory diseases i.e., (psoriasis,arthritis, Sjoegren's syndrome, inflammatory bowel disease) that may affect levels of salivary cytokines were excluded.

Statistical Analysis: SPSS software 22 was used for statistically analysis. The data are expressed as mean \pm SD. Significance of the difference between the mean value of the measured parameters between groups were evaluated by Student's t-test. And chi-square. Correlation was indicated by Pearson correlation tests and $\mathrm{P}<0.05$ is considered significant. 


\section{Result}

Themean BMI, age, and sex between the experimental group and the control group were similar, were not statistically significant $(\mathrm{p}=0.466, \mathrm{p}=0.240, \mathrm{p}=0.455)$, respectively while, there were a significant difference in CRP between the two groups $(\mathrm{p}=0.037)$. Concentrations of salivary IL-6, IL-8 and TNF- $\alpha$ were not affected by periodontal health. No statistically significant difference in periodontal health, assessed by CPITN, were observed between the groups $(p=0.413)$ [Table 1]. Salivary levels of IL-6 and IL-8 were significantly higher in smokers with dental caries compared with $\operatorname{control}(p<0.05)$. No significant differences in salivary TNF- $\alpha$ in dental caries patients compared to control $(\mathrm{p}=0.063)$ [Table2]. The correlation between TNF- $\alpha$, CRP, age, sex, and BMI with IL-8 were positive and statistically significant $(\mathrm{p}=0.01$, $\mathrm{p}=0.036, \mathrm{p}=0.009, \mathrm{p}=0.007$, and $\mathrm{p}=0.005$ ), respectively. There was no significant correlation between IL-6 and TNF- $\alpha$ cytokines and other parameters, except for a positive association with CRP levels $(\mathrm{p}=0.006, \mathrm{p}=0.043)$, respectively [Tab 3].

Table 1. Comparison between dental caries patients and controls according to BMI, age, sex, CRP, and CPITN.

\begin{tabular}{|c|c|c|c|}
\hline $\boldsymbol{p}$-Value & Control $($ Mean \pm SD) & Dental Caries cases (Mean \pm SD) & Parameters \\
\hline 0.466 & $23.14 \pm 5.22$ & $22.33 \pm 8.02$ & BMI $\left(\mathrm{kg} / \mathrm{m}^{2}\right)$ \\
\hline 0.240 & $41.76 \pm 3.41$ & $46.78 \pm 4.36$ & Age $($ years) \\
\hline 0.455 & $29.17 \pm 5.88$ & $28.34 \pm 7.06$ & Sex \\
\hline $0.037^{*}$ & $3.14 \pm 2.16$ & $9.87 \pm 2.55$ & CRP $(\mathrm{mg} / \mathrm{L})$ \\
\hline 0.413 & $2.18 \pm 1.05$ & $2.33 \pm 1.12$ & CPITN \\
\hline
\end{tabular}

CPITN: Community Periodontal Index of Treatment Needs * $\mathrm{p}<0.05$ :Differences between patients with dental caries and healthy group

Table 2. Salivary interleukin 6 , interleukin 8 , and tumor necrosis factor alpha in patients with dental caries and controls.

\begin{tabular}{|c|c|c|c|}
\hline $\boldsymbol{p}$-Value & Control(n=16) $($ Mean \pm SD) & Dental Caries Cases(n=32) (Mean \pm SD) & Cytokines \\
\hline $0.005^{*}$ & $2.58 \pm 5.56$ & $43.88 \pm 28.76$ & $\mathrm{IL}-6(\mathrm{pg} / \mathrm{mL})$ \\
\hline $0.008^{*}$ & $616.15 \pm 309.33$ & $1597 \pm 957.22$ & $\mathrm{IL}-8(\mathrm{pg} / \mathrm{mL})$ \\
\hline 0.063 & $8.20 \pm 5.85$ & $8.54 \pm 5.88$ & $\mathrm{TNF}-\alpha(\mathrm{pg} / \mathrm{mL})$ \\
\hline
\end{tabular}

$* \mathrm{p}<0.05$ : Differences between patients with dental caries and healthy group.

Table 3. Correlation between proinflammatory cytokines and C-reactive protein, BMI, age and sex in dental caries patients.

\begin{tabular}{|c|c|c|c|c|c|c|c|}
\hline BMI & Sex & Age & CRP & TNF- $\alpha$ & IL-8 & IL-6 & Parameters \\
\hline 0.091 & 0.122 & 0.083 & $0.006^{*}$ & 0.332 & 0.877 & - & IL-6 \\
\hline $0.005^{*}$ & $0.007^{*}$ & $0.009^{*}$ & $0.036^{*}$ & $0.01^{*}$ & - & 0.877 & IL-8 \\
\hline 0.410 & 0.344 & 0.225 & $0.043^{*}$ & - & $0.01^{*}$ & 0.332 & TNF- $\alpha$ \\
\hline
\end{tabular}

$* \mathrm{p}<0.05$ : Differences between smokers with dental caries and healthy group.

\section{Discussion}

Cytokines regulate many aspects of the immune response, therefore, along with other factors, they will be useful tools for diagnosing and monitoring the oral cavity, and saliva can be used as diagnostic material to measure biomarkers released during disease onset and progression. IL-6, IL-8, and TNF- $\alpha$ immune cell products play an important role in oral mucosa diseases, but the exact role of cytokines in the etiology of dental caries is not well known. In the innate response, the 
molecular phenotypes associated with oral pathogens bind to the receptors of the host cells, including dendritic cells, which then activate the inflammatory response by releasing the active of pro-inflammatory cytokines, i.e., IL-1b, IL-6, IL-8 and TNF- $\alpha^{(3)}$. Initially, gingivitis may only develop, and if the immune response is successful, periodontitis will resolve;however, if the infection persists and more bacteria spread occurs, intensication the warning response can damage gum tissue in infected subjects $^{(13)}$. The mean BMI, age, and sex between the experimental group and the control group were similar, were not statistically significant $(\mathrm{p}=0.466, \mathrm{p}=0.240$, $\mathrm{p}=0.455$ ), respectively. while, there were a significant difference in CRP between the two groups $(\mathrm{p}=0.037)$. Concentrations of salivary IL-6, IL- 8 and TNF- $\alpha$ were not affected by periodontal health.No statistically significant difference in periodontal health, assessed by CPITN, were observed between the groups $(p=0.413)$ [Table 1]. These results are consistent with other studies $^{(1,14)}$. One study indicated that elevated CRP levels were a risk factor for developing one type of cancer. High levels of CRP before surgery are an unwanted diagnostic indicator ${ }^{(15)}$. Yadav and Prakash indicated that the pattern of dental caries varies with BMI, sex, age, race, food habits, socio economic status, geographical location and oral hygiene practices ${ }^{(16)}$. There is a correlation between CRP levels and disease prognosis.In the study by Park et al., an increased CRP/ albumin ratio was correlated with prolonged disease and decreased survival rate ${ }^{(17)}$. Blatt et al. noted that CRP, ferritin, and hemoglobin can be used as biomarkers in diagnosis and disease progression ${ }^{(18)}$. The same idea is supported by Tai et al. in patients with oral squamous cell carcinoma (OSCC), which they showed a positive association between high CRP levels and oral cancer, and revealed that CRP level was associated with invasion of localized lymph nodes ${ }^{(19)}$. Relationship between cancer and inflammation is bidirectional. The tumor triggers an inflammatory response by increasing serum levels of CRP on the one hand, and chronic inflammation is involved in the development of a malignant process, on the other hand ${ }^{(3)}$.On the other hand, Grimm et al. identified high levels of CRP, high number of total leukocytes, neutrophils, monocytes; and a low number of lymphocytes is correlate with a low survival rate in oral cancer ${ }^{(20)}$. Salivary levels of IL-6 and IL-8 were significantly higher in smokers with dental caries compared with control $(p<0.05)$ [Table 2]. The levels of salivary cytokines are elevated as a result of disturbances in the oral cavity, and these results are in line with previous studies ${ }^{(13,14)}$. Also, these results were confirmed by the Kurtis et al. where they found that high levels of IL-6 in dental caries patients lead to fewer broblasts and osteoblasts. It also supports demineralization of teeth and the development of cavities, especially in smokers ${ }^{(21)}$. IL-8 play an important role in the acute inflammatory response and persists for a relatively long time at the site of inflammation ${ }^{(3)}$. Garrido et al.showed significantly higher levels of CRP and IL-6 in subjects diagnosed with apical periodontitis compared to normal healthy teeth $^{(22)}$. Tumor can trigger an inflammatory response, triggering the release the cytokines i.e., IL-1 $\beta$ and IL-6. Márton et al. indicated that the specificity and sensitivity of IL-6 protein were less favorable than IL-6 mRNA, and the expression of salivary IL-6 mRNA was significantly associated with dental status and age ${ }^{(23)}$. On the other hand, Gabay reported that interleukin-6, in addition to the presence of other cofactors, causes resorption of bones and stimulates synthesis of chemocines $^{(6)}$. Saheb Jamee et al. found significantly higher concentrations of IL- 6 in patients with OSCC and in the saliva of chronic periodontitis compared toIL- 8 and TNF-olevels ${ }^{(24)}$.Gornowicz et al. reported significantly elevated salivary IL-6 levels in children with dental caries. Consequently, the researchers speculated that the dysfunction of the triangle oral mucosa, immune cells and saliva lead to the emergence of oral mucosa diseases i.e., periodontitis, leukoplakia, oral lichen planus and oral cancer ${ }^{(1)}$. Our results differ from the Teles et al., on the one hand, as they reported lower levels of IL-8 and elevated levels of TNF- $\alpha^{(25)}$. On the other hand, it is compatible, as they reported elevated levels of IL-6 in a healthy periodontally group compared with chronic periodontitis. IL-6 receptor stimulates the activation of signal transducers, Janus kinases (JAK), and activators of transcription (STATs), which then stimulate pathways that include mitogenactivated protein kinase (MAPK), which in turn supports cancer development, This may explain the reasons for the elevated levels of IL- 6 in the saliva of smokers with dental caries and OSCC patients. Manifestations of IL-6 have been detected in tumor cells and tumor-infiltrating leukocytes, and this indicates the presence of a paracrine loop of stimulation. Colak et al., show that children who are frequently exposed to sugary fluids, formula milk, breast milk, fruit juices, etc. from sweet liquids for long periods are at risk of early childhood caries(ECC) ${ }^{(26)}$. Consequently, Menon et al. concluded that the problems that children face especially ECC are not just pain and infection, but they also affect communication, speech, 
eating, dietary nutrition, playing, etc ${ }^{(27)}$. No significant differences in salivary TNF- $\alpha$ in dental caries patients compared to control $(\mathrm{p}=0.063)$ [ Table 2] .These results are consistent with other studies ${ }^{(7,28)}$.Contrary to our findings, Kurtis et al. found that high levels of TNF- $\alpha$ in the saliva of dental caries patients lead to fewer broblasts and osteoblasts, and aid in demineralization of teeth and the development or deepening of cavities, especially in smokers $^{(21)}$. One study showed that TNF- $\alpha$, which is produced by osteoclasts, is an important factor that regulates the spacing of these cells as well as is involved in the processes of resorption ${ }^{(29)}$. Ghallab et al., found a lower elevation of TNF- $\alpha$ in erosive oral lichen planus (OLP) patients after treatment ${ }^{(30)}$. Mclachlan et al., conclude that pro-inflammatory cytokine concentrations are also elevated within both gingival tissues and serum of persons with periodontal inflammation ${ }^{(31)}$. The DEATH protein bound to TNF- $\alpha$ is encoded by the TRADD gene, which recruits TNF- $\alpha$ receptor-associated factor-2, a protein is encoded by the TRAF2 gene and signaling molecule RIP which activates and stimulates the Nuclear Factor Kappa-light-chainenhancer of activated $\mathrm{B}$ cells (NF- $\mathrm{KB}$ ) pathway, and is involved in cell survival and proliferation and anti-apoptotic agents, and this explains why TNF- $\alpha$ levels did not change in patients' saliva. The correlation between TNF- $\alpha$, CRP, age, sex, and BMI with IL-8 were positive and statistically significant $(\mathrm{p}=0.01, \mathrm{p}=0.036, \mathrm{p}=0.009$, $\mathrm{p}=0.007$, andp $=0.005$ ), respectively [Tab3]. These results have been confirmed by other studies ${ }^{(1,13)}$.IL-8 is released by neutrophils and macrophages in response to various stimuli i.e., steroids, inflammatory signals, chemical environment, and environmental stresses that activate the nuclear factor-kappa-B(NF- $\mathrm{B}$ ) pathway that activates the expression production of IL-8. IL-8 and TNF- $\alpha$ play an important role in immunity of the oral cavity. It is possible that elevated levels of IL-8 lead to stimulation of TNF- $\alpha$. These results have been supported by previous studies ${ }^{(1,13)}$ IL- 8 levels may change with lifestyle, ethnic differences, geographic distribution, genetic differences, and individual habits. Elevated IL-8 levels may be found in gingivitis and periodontitis. However, none of these cases can raise the levels to the extent seen in oral cancer ${ }^{(7,32)}$.Punyani and Sathawane found that increased levels of IL- 8 confirmed its role in angiogenesis and progression ${ }^{(32)}$. Therefore, large changes in IL-8 levels provide us with great potential for use as a single salivary biomarker.This could open new horizons for treatment plans from targeted leukotriene therapy ${ }^{(31)}$.
There were no significant correlation between IL-6 and TNF- $\alpha$ cytokines and other parameters, except for a positive association with CRP levels $(p=0.006, p=0.043)$, respectively [Tab 3].This conclusion was confirmed by a previous report ${ }^{(3)}$.Sugawara indicated that TNF- $\alpha$ is an effective cellular compound and its production leads to oral diseases ${ }^{(32)}$.This means that the salivary level of cytokines may be increased as a result of disorders in the oral cavity. These results are consistent with other studies ${ }^{(7,33)}$.Barnes et al., reported that IL-6 can be generated not only by activated macrophages/ monocytes,but also by fibroblasts and activated endothelial cells in inflamed tissue,while TNF- $\alpha$ is produced predominantly by activated macrophages at the site of infection/inflammation, and also by T cells.,In turn,IL-6 production can lead to the inhibition of TNF- $\alpha$ expression $^{(36)}$.This finding supports our current results. On the other hand,Li et al. indicated that up-regulation of MUC1 expression in oral epithelial cells result from Porphyromonas gingivalis infection or increases in pro-inflammatory cytokines such as IL-1 $\beta$, IL-6, and $\mathrm{TNF}-\alpha^{(37)}$. Contrary to our results,Fine et al., reported in their study that dental caries was not significantly correlated with concentrations of IL-1 $\beta$, IL-1ra, or IL10 in patients' serum and saliva ${ }^{(38)}$.Several studies have indicated the pivotal role of chronic inflammation in carcinogenesis by modifying inflammatory cells and cytokine production ${ }^{(3,37)}$.This explains why cytokine levels are high in patients, and thus support our current findings. Also, the result of this study confirmed by Barnes et al.,who stated that IL-6 can be generated not only by activated macrophages/monocytes, but also by fibroblasts and activated endothelial cells in inflamed tissue, while TNF-a is known to be produced predominantly by activated macrophages at the site of infection/inflammation, and also by $\mathrm{T}$ cells ${ }^{(36)}$.In turn,IL-6 production can lead to the inhibition of TNF- $\alpha$ expression $^{(39)}$. SahebJamee et al. found significantly higher concentrations of IL- 6 in patients with OSCC and in the saliva of chronic periodontitis compared to cytokine levels (IL-6, 8, and TNF- $\alpha)^{(24)}$.Data from this study indicate links between the production of inflammatory, and immunoregulatory cytokines and chemokines(IL-6, IL-8 and TNF- $\alpha$ ), and dental caries in smokers. Therefore these parameters can be used as biomarkers to indicate disease severity and progression.

\section{Conclusions}

The results indicate links between production of IL-6 and IL-8 in smokers' saliva and dental caries. 
Excretion of specific cytokines in patients' saliva are useful tools for diagnosing and monitoring dental caries disease. Therefore, can be used saliva as a noninvasive diagnostic fluid to measure biomarkers released during disease onset and progression.

Acknowledgment. The authors are grateful to all the patients and healthy subjectes who participated in this study. Also, the authors are grateful to the faculty in Preventive Dentistry, Department of Pedodontics, and Department of Biochemistry for their valuable support towards this clinical research.

Ethical clearance. Ethical clearance taken from AlSaddr Teaching Hospital committee in Basra city,Iraq.

Financial support and sponsorship.Nil. Conflicts of interest.There are no conflicts of interest.

\section{References}

1. Gornowicz A,Bielawska A,Bielawski $\mathrm{K}$ et al. Proinflammatory cytokines in saliva of adolescents with dental cariesdisease. Ann Agric Environ Med 2012;19(4):711-716.

2. Thean H,Wong ML,Koh H. The dental awareness of nursing home staff in Singapur- a pilot study. Gerodontology2007; 24: 58-63.

3. Tampa M,Mitran MI,Mitran CI et al. Mediators of Inflammation- A Potential Source of Biomarkers in Oral Squamous Cell Carcinoma, J Immunology Research, 2018, Article ID 1061780,12 pages.

4. Zhang JM and An J.Cytokines, inflammation, and pain, International Anesthesiology Clinics2007; 45(2): 27-37.

5. Surcel M,Constantin C,Caruntu $\mathrm{C}$ et al.Inflammatory cytokine pattern is sex-dependent in mouse cutaneous melanoma experimental model, JImmunology Research, 2017, Article ID 9212134,10 pages.

6. Gabay C. Interleukin-6 and chronic in-ammation. Arthritis Reser.2006; 8(2): S3-S6.

7. Sahibzada HA, Khurshid Z,Khan RS et al .Salivary IL-8, IL-6 and TNF- $\alpha$ as Potential DiagnosticBiomarkers for Oral Cancer. Diagnostics2017; 7(21):2-10.

8. DePaepe B,Creus KK,De Bleecker JL. The tumor necrosis factor superfamily of cytokines in the in-ammatory myopathies:Potential targets for therapy.Clin Dev Immunol.2012;2012:Article ID
369462.

9. Slattery ML, Lundgreen A, Bondurant KL et al. Tumor necrosis factor-related genes and colon and rectal cancer. Int J Mol Epidemiol Genet. 2011; 2(4):328-338.

10. StJohn MA,Li Y,Zhou X et al. Interleukin 6 and interleukin 8 as potential biomarkers for oral cavity and oropharyngeal squamous cell carcinoma.Arch. Otolaryngol. Head. Neck Surg.2004;130, 929-935.

11. Wu-Wang CY,Patel M,Feng J et al. Decreased levels of salivary prostaglandin E2 and epidermal growth factor in recurrent aphthous stomatitis.Arch Oral Biol.1995; 40:1093-8.

12. Ainamo J,Barmes D,Beagrie $G$ et al. Development of the World Health Organi- zation(WHO) community periodontal index of treatment needs (CPITN).J Int Dent 1982;32:281-91.

13. Giudice GL,Nicita F,Militi A et al. Correlation of s-IgA and IL-6 Salivary with Caries Disease and Oral Hygiene Parameters in Children .J Dent2020; 8, 3:2-7.

14. Peres CD, Gava RP,Zen IR et al. Salivary levels of Interleukin-6 and Tumor Necrosis Factor-alpha in girls aged7---17years practicing volleyball.Apunts Med. Esport. 2019;54 (203): 91-101.

15. Khandavilli SD,Ceallaigh PÓ,Lloyd CJ et al.Serum C-reactive protein as a prognostic indicator in patients with oral squamous cell carcinoma,Oral Oncology2009; 45(10): 912-914.

16. Yadav k,Prakash S.Dental Caries:A Review,Asian JBiomedical and Pharmaceutical Sciences. Nepal,Clin Oral Invest2016; 8:91-96.

17. Park HC,Kim MY,Kim CH. C-reactive protein/ albumin ratio as prognostic score in oral squamous cell carcinoma,J Korean Association of Oral and Maxillofacial Surgeons 2016;42(5): 243-250.

18. Blatt S,Schön H,Sagheb K et al. Hemoglobin,Creactive protein and ferritin in patients with oral carcinoma and their clinical significance a prospective clinical study,J Cranio-Maxillofacial Surgery 2018;46(2):207-212.

19. Tai SF,Chien HT,Young CK et al.Roles of preoperative $\mathrm{C}$-reactive protein are more relevant in buccal cancer than other subsites, World JSurgical Oncology2017;5(1): 47.

20. Grimm M,Rieth J,Hoefert S et al. Standardized pretreatment inflammatory laboratory markers and 
calculated ratiosin patients with oral squamous cell carcinoma, European Archives of Oto-RhinoLaryngology 2016; 273(10): 3371-3384.

21. Kurtis B,Tuter G,Serdar $M$ et al. Gingival crevicularuid levels of monocyte chemoattractant protein-1 and tumor necrosis factor- $\alpha$ in patients with chronic and aggressive periodontitis. J Periodontol 2005; 76(11):1849-1855.

22. Garrido M,Dezerega A,Bordagaray $M$ et al. C-reactive protein expression is upregulated in apical lesions of endodontic origin in association with interleukin-6.J Endod 2015;41:464-9.

23. Márton IJ,Horváth J,Lábiscsák P et al. Salivary IL-6 mRNA is a Robust Biomarker in Oral Squamous Cell Carcinoma.J Clin.Med.2019;8,1958.

24. SahebJamee M,Eslami M, AtarbashiMoghadam F et al. Salivary concentration of TNF-a,IL-1-a,L-6 and IL-8 in oral squamous cell carcinoma.Med Oral,Patol Oral Cir Bucal.2008;13(5):E292-E295.

25. Teles R,Sekellari D,Teles F et al. Relationships among gingival crevicularuid biomarkers,clinical parametres of periodontal disease,and the subgingival microbiota.J Periodontol. 2010; 81(1): 89-98.

26. Colak H,Dülgergil C,Dalli M et al. Early childhood caries update: A review of causes, diagnoses, and treatments. JNat Sci Biol Med2013;4:29-38.

27. Menon MM,Malagopal RV,Sajitha $\mathrm{K}$ et al.Evaluation of salivary interleukin-6 in children with early childhood caries after treatment. Contemp Clin Dent.2016;7(2):198-202.

28. Brailo V, Vucicevic-Boras V,Lukac $\mathrm{J}$ et al. Salivary and serum interleukin 1beta, interleukin 6 and tumor necrosis factor alpha in patients with leukoplakia and oral cancer. Med Oral Patol Oral Cir Bucal. 2012;17(1):10-5.

29. Tani-Ishii N,Tsunoda A,Teranaka T et al.Autocrine regulationof osteoclast formation and bone resorption by IL-1 alpha and TNF alpha. J Dent Res.1999;78(10): 1617-1623.
30. Ghallab NA,El-Wakeel N,Shaker OG. Levels of salivary interferongamma,TNF-a, and TNF receptor 2 as prognostic markers in erosive lichen planus.Mediators In-amm. 2010; 2010: Article ID 847632.

31. McLachlan JL, Sloan AJ,Smith AJ et al. S100 and Cytokine Expression in Caries Infect Immun. 2004;72(7):4102-4108.

32. Punyani SR,Sathawane RS.Salivary level of interleukin-8 in oral precancer and oral squamous cell carcinoma,Clinical Oral Investigations 2013;17(2):517-524.

33. Lee $\mathrm{S}$ and Margolin K.Cytokines in cancer immunotherapy. Cancers 2011,3,3856-3893.

34. Sugawara S.Host defence of oral mucosa and the molecular mechanismof oral mucosal signal transduction diseases. J Oral Biosci. 2005; 47:115125.

35. Singh P,Verma JK, Singh J.Validation of Salivary Markers,IL-1 $\beta, I L-8$ and Lgals3bp for Detection of Oral Squamous Cell Carcinoma in an Indian Population. Scientific Report S 2020;10:7365.

36. Barnes TC,Anderson ME, Moots RJ. The many faces of interleukin-6: The role of IL-6 in inflammation, vasculopathy, and fibrosis in systemic sclerosis.Int JRheumatol. 2011; 2011: Article ID 721608.

37. Li X,Wang L,Nunes D et al. Pro-inflammatory cytokines up regulate MUC1gene expression in oral epithelial cells.J DentRes 2003; 82:883-87.

38. Fine DH,Markowitz K,Furgang D et al.Macrophage inflammatory protein-1 alpha:a salivary biomarker of bone loss in a longitudinal cohort study of children at risk for aggressive periodontaldisease? J Periodontol 2009;80(1):106-113.

39. Georgescu SR,Ene CD,Nicolae I L. Reflectometric analysis for identification of various pathological conditions associated with lichen planus, Revista de Chimie 2017,68(5):1103-1108. 